\title{
Marc GOOSSENS
}

\section{A strong token of interest for an excellent book about Life Long Learning}

For anyone who is involved in Lifelong Learning and wants to get a deep understanding of why and how it fits the present and near future evolution of our knowledge society, Anikó KÁLMÁN's book "Learning - in the New Lifelong and Lifewide Perspectives " ${ }^{1}$ is an absolute must.

I have been involved in Continuing Engineering Education for some twenty years and I have known Anikó for about half that time, so, though I knew that her book would be interesting, I was curious to read it. I have to admit, not only that it brought to me lots of 'things' I did not know of, but also that it opened new perspectives for further thoughts.

Jointed in three main chapters, this book can be considered as a system composed of two adjacent rooms (the first and the third chapters) linked by a sort of one-way door (the second chapter):

- The first - and longest - chapter describes the "state of learning" in the first room - which most of us are still living in - and why a change of paradigm is necessary, driven by the impact of globalization and new technologies upon the fabric of society. In this long chapter, the author, in line with the title of her book, presents a perspective of learning as an activity, both along a time axis ( historically speaking) and a space axis ( with its different systems and sub-systems ).

- The second chapter explains the mechanism thanks to which the door linking the two rooms could be opened: a more social innovation process, for which the Knowledge Triangle acts as a conceptual key. In the same way that a door is thinner than a room, this short chapter is just a suggestion of how to push on the handle and open the door.

- And the third chapter describes the "state of learning" that we could expect to achieve in the second room, mainly the relationship between different types of teachers and different types of learners. Although this second room is gigantic, this third chapter is also very short, because we are blind as regards the future, we can only discover it gradually as we build it ; and there is no single way to build our future.

From the beginning of the first chapter, as the author introduces the three main values of modern society and opposes eco-philosophy to the present philosophy, the reader will understand that he or she is bound for a fundamental approach of lifelong learning, considered here as a basic element of our knowledge society.

To that respect, considering - as the author does - that lifelong learning has also to be a lifewide learning, in other words that it is not only the continuation of a learning activity along a temporal

${ }^{1}$ Edited by Tampere University of Applied Sciences, ISBN 978-952-5903-80-5, 2016 
linear direction, but that it also comprises - as any educational process - a widening of the 'socle' upon which higher and higher levels of knowledge and understanding can become integrated in a harmonized way, is in my opinion an important - the most important indeed - contribution of this book.

The author shows that, as a result of this reinforcement process, the individual can pass from conformist learning to successively conscientious and autonomous learning ( the temporal axis ) and integrate nonformal and informal education besides formal education ( the space direction ). After that, in what forms the main part of her book, the authors analyses the various implications of this premise for all the aspects of learning - and therefore, also, of teaching - from a systemic point of view: learning and teaching become some sort of merged activity, the objective of which is not the individual, but the society in which this one is living. Therefore, the two main challenges are, on the one hand the transmission of knowledge and know-how from one generation to the next one, and on the other hand - as a consequence of the first challenge - see to it that the individual possesses a high enough level of skills to efficiently transfer his or her individual knowledge and know-how to society.

In the second and third chapters, the author considers more practical - and sometimes psychological - implications of the previous theoretical approaches, one being the necessity to link education and research to the needs of society ( the motivation counterpart of those activities ), and the other one being the requirements that have to be met for developing self-directed learning.

As a physicist, I know that all things in our universe are governed by the same laws, that we stand somewhere between the infinitely great and the infinitesimal, and that we cannot understand one if we don't understand the other one.

As an engineer, I know that, if I have to build some 'system', the environment in which this system is going to work is at least as important as the composition and the working of the system itself.

As a physicist and engineer having developed some expertise in Continuing Engineering Education, I deem education to be a living and extraordinary complex system that can efficiently serve society if - and only if - it takes into account both the needs of society and the requirements and limitations of its constitutive parts.

This is why I consider the concepts and viewpoints that are tackled in this book as being of utter importance to anyone involved in education, particularly in Higher Education and in Lifelong Learning, but also to all professionals who have to regularly update or widen their competencies. This is particularly true for engineers, as they work in a fast evolving sector and must resort to Continuing Engineering Education.

I can do nothing but prompt them to read Anikó KÁLMÁN’s book. 